\title{
Association between Vitamin D Level in Blood and Periodontitis in Korean Elderly
}

\author{
Na-Na Yoon, Ji-Young Lee ${ }^{1 \dagger}$, and Byeng-Chul $\mathrm{Yu}^{2}$ \\ Kosin University Graduate School, Busan 49267, \\ ${ }^{1}$ Department of Dental Hygiene, Jinju Health College, Jinju 52655, \\ ${ }^{2}$ Department of Preventive Medicine, College of Medicine, Kosin University, Busan 49267, Korea
}

\begin{abstract}
This study identified an effective control method for periodontitis by investigating the association between blood levels of vitamin $\mathrm{D}$ and periodontitis in Korean elderly based on raw data from the fifth Korea National Health \& Nutrition Examination Survey of 2010 (KNHANES). In this study, 1,021 adults over 65 years of age were evaluated based on data from the KNHANES. Periodontal disease was assessed using community periodontal index (CPI), with CPI codes $\geq 3$ defined as periodontitis. Blood levels of vitamin D were measured from blood samples and divided into four groups (first quartile: $\leq 13.23 \mathrm{ng} / \mathrm{ml}$, second quartile: $13.24 \sim 16.95 \mathrm{ng} / \mathrm{ml}$, third quartile: $16.96 \sim 21.58 \mathrm{ng} / \mathrm{ml}$ ), and fourth quartile $>21.59 \mathrm{ng} / \mathrm{ml}$ ). Using multiple logistic regression analyses, the variables were adjusted for general characteristics, oral health-related characteristics, health-related characteristics, and bone mineral density. The statistical analysis was performed using the SAS (ver. 9.2). The results of this study are as follows: the prevalence of periodontitis was $42.6 \%$ in Korean elderly. After adjusting for general, oral health-related, and health-related, the risk of periodontitis in the first quartile group was 1.74 times (95\% confidence interval [Cl], 1.02 2.98) higher than that of the fourth quartile group $(p=0.041)$. After adjusting for general, oral health-related, and health-related characteristics as well as bone mineral density, the risk of periodontitis in the first quartile group was 1.73 times $(95 \% \mathrm{Cl}, 1.02 \sim 2.96)$ higher than that of the four quartile group $(\mathrm{p}=0.042)$. There was a significant relationship between blood vitamin D level and periodontitis in Korean elderly. For the prevention of periodontitis, factors related to vitamin D should be considered along with other risk factors.
\end{abstract}

Key Words: Bone density, Elderly, Korean, Periodontitis, Vitamin D

\section{Introduction}

Periodontal disease is a chronic inflammatory condition caused by various factors, including hereditary, local or systematic factors, in addition to pathogenic bacterial factors. In the earliest stage, the inflammation forms only on the gingiva (or gums); however, as it progresses, the depth of the gum pocket increases and the tooth loosens due to periodontal tissue loss and alveolar bone resorption. It is a common oral disease that leads to tooth loss in adults. According to the Korea National Health \& Nutrition Examination Survey (KNHANES), the prevalence of periodontitis among adults over 19 years of age was $22.7 \%, 27.7 \%$, and $29.2 \%$ in 2012,2013 , and 2014 , res- pectively whereas the prevalence of periodontitis among elderly people over 65 years of age was $42.4 \%, 48.5 \%$, and $44.9 \%$, respectively ${ }^{11}$.

Vitamin D is a crucial substance that aids in bone growth and retention and maintains the homeostasis of minerals ${ }^{2}$. Vitamin D deficiency leads to decreased calcium absorption in the small intestine and kidneys by $10 \sim 15 \%$, subsequently lowering blood calcium levels. To compensate for this deficiency, the secretion of parathyroid hormone increases. Chronic vitamin D deficiency causes decreased blood calcium levels and increased levels of parathyroid hormone, which activates $1,25(\mathrm{OH}) \mathrm{D}$ and osteoclasts. To compensate for deficient blood calcium levels, calcium is released from the calcified bone matrix, 
which prevents bone calcification, decreases bone mineral density, and increases the risk of osteoporotic bone fracture $^{3)}$. Periodontal disease is mainly characterized by the loss of connective tissue and alveolar bone; under the premise that decreased bone mineral density accompanies the loss of alveolar bone, studies have reported the correlations between osteoporosis and alveolar bone resorption ${ }^{4)}$, between overall bone mineral density and alveolar bone height ${ }^{5,6)}$, and between bone mineral density and tooth loss ${ }^{7-11)}$. A side from regulating calcium homeostasis, vitamin $\mathrm{D}$ is also involved in immunity by binding cellular vitamin $\mathrm{D}$ receptors, converting to $1,25(\mathrm{OH})_{2} \mathrm{D}_{3}$ as it moves into immunocytes such as microphages and dendritic cells ${ }^{12)}$. Vitamin D also exertspositive effects on periodontal health: higher blood levels of vitamin D reduce gingival bleeding ${ }^{13)}$ and increase the antibacterial activity of oral gingival epithelial cells ${ }^{14)}$.

Bone mass is established through the process of the formation, resorption, and reformation of bone tissues; in old age, bone resorption occurs more frequently than bone formation, leading to reduced bone mass ${ }^{15)}$. Particularly after menopause, women experience a rapid bone loss due to the effect of hormonal changes ${ }^{16)}$. In regard to bone health, vitamin D plays a significant role in affecting bone growth or retention and bone mineral density.

Vitamin D deficiency in the elderly increases the risk of various diseases, including osteoporosis, osteopenia, and cardiovascular diseases ${ }^{17)}$. Due to indoor life styles, loss of appetite, and chronic diseases, it is easy to become deficient in vitamin $\mathrm{D}^{18)}$. Clinical diseases that have been associated with vitamin D deficiency were identified mainly through epidemiological studies, but most did not adjust for disruption variables such as diet, everyday vitamin D levels, body mass index, age, or physical activity ${ }^{19,20)}$. In addition, many research subjects in previous studies were post-menopausal women ${ }^{5,7,9)}$. While studies have assessed the correlation between bone mineral density and periodontal diseases and the correlation between vitamin $\mathrm{D}$ and bone tissue, few have focused on the association with periodontal diseases by considering vitamin $\mathrm{D}$ and bone mineral density.

In this context, the present study was conducted to provide baseline data for the prevention and treatment of periodontitis by investigating the correlation between blood levels of vitamin D level with bone mineral density and periodontitis among elderly people over 65 years of age and by identifying the risk factors for periodontitis.

\section{Materials and Methods}

\section{Subjects}

The present study used raw data from the first-year survey of the fifth Korea National Health and Nutritional Examination Survey (KNHANES). The first survey was conducted from January to December 2010. A total of 8,058 people participated in more than one form of survey among the health, health examination, and nutrition surveys. To investigate the association between periodontal diseases and bone mineral density, this study selected 1,021 elderly people over 65 years of age whose periodontal tissue, bone mineral density, and blood vitamin $\mathrm{D}$ level were measured in response to dental exam and health survey questions. Prior to this study, the researcher obtained approval from the Institutional Review Boards at Kosin University Gospel Hospital (KUGH2015-10-005-002).

\section{Methods}

The independent variables of this study included socio-demographic characteristics (five questions), oral health behavior characteristics (four questions), general health behavior characteristics (five questions), blood vitamin $\mathrm{D}$ level, and bone mineral density. The dependent variable was the periodontitis status as determined during the dental exam.

\section{1) Blood vitamin D levels}

In addition tothe health examination questions, blood levels of vitamin D level were measured in regular blood tests and divided into four quartile groups as follows: first quartile (Q1), vitamin D level $\leq 13.23 \mathrm{ng} / \mathrm{ml}$; second quartile (Q2), $13.24 \sim 16.95 \mathrm{ng} / \mathrm{ml}$; third quartile (Q3), $16.96 \sim 21.58 \mathrm{ng} / \mathrm{ml}$; and fourth quartile (Q4), > 21.59 $\mathrm{ng} / \mathrm{ml}(\mathrm{Q} 4)$. Because there is no consensus optimal blood vitamin D level, this study divided the vitamin D levels into quartile groups in order to examine these levels in 
elderly people in the KNHANES, to identify problems with vitamin D levels, and to determine the optimal level for the prevention of periodontitis based on previous study findings.

\section{2) Bone mineral density}

The subjects were divided based on overall femoral T-scores from bone mineral density tests collected as part of the health exam items. The t-score was the number derived from dividing the difference between participant bone mineral density score and the highest bone mineral density score of a young adult in the same sex group by standard deviation. $\mathrm{T} \geq-1.0$ was categorized as normal range; $-1.0>\mathrm{T}>-2.5$ and $\mathrm{T} \leq-2.5$ were considered osteopenia and osteoporosis, respectively ${ }^{21)}$.

\section{3) Periodontitis}

The condition of the periodontal tissue was assessed using the community periodontal index (CPI). After dividing the oral cavity into sextants (1st and 2nd maxillary molars on the left, maxillary central incisor, 1st and 2nd maxillary molars on the right, 1 st and 2 nd mandibular molars on the left, mandibular central incisor, and 1st and 2nd mandibular molars on the right), the researcher measured around the 10 teeth for standard testing in terms of the bleeding status, plaque attachment, and depth of the periodontal pocket using the periodontal probe designed by the World Health Organization. The highest score of the sextants was recorded. Normal periodontal tissue was healthy tissue (CPI 0); bleeding gum on probing was considered bleeding periodontal tissue (CPI 1); periodontal tissue with plaque attachment was considered periodontal tissue with plaque retention (CPI 2); periodontal tissue with a $4 \sim 5 \mathrm{~mm}$ gum pocket depth was considered shallow pocket periodontal tissue (CPI 3); and periodontal tissue with over $6 \mathrm{~mm}$ periodontal pocket depth was considered deep pocket periodontal tissue (CPI 4). Based on the clinical definition of periodontist for CPI values above $3^{22}$, this study also classified subjects with a CPI value 3 or above in one of the sextants as having periodontitis.

\section{Data analysis}

With regard to the data from the KNHANES, a complex sampling analysis method that utilizes cluster sampling variables and estimated variance with individual weight was employed to maintain the rolling survey sampling method and perform a more accurate data analysis.

Frequency and cross over analyses were performed on variables such as sociodemographic characteristics, health behavior, oral health behavior, periodontitis status, blood vitamin D level, and bone mineral density. To examine the risk of periodontitis, multiple logistic regression analysis was performed on independent variables by adjusting for the sociodemographic characteristics, health behavior characteristics, oral health behavior characteristics, and bone mineral density. The data were analyzed using SAS (ver. 9.2; SAS Institute, Cary, NC, USA) and tested for significance at $\mathrm{p}<0.05$.

\section{Results}

\section{The prevalence of periodontitis based on research subjects sociodemographic characteristics}

Examination of the prevalence of periodontitis based on the research subjects' general characteristics showed the following: by sex, periodontitis was more prevalent among men $(46.5 \%)$ than women $(41.5 \%)$; by education level, periodontitis was most prevalent among subjects with a middle school education $(51.5 \%)$; by income level, periodontitis was most prevalent among the low middle class $(44.4 \%)$; by area of residence, periodontitis was more prevalent in rural areas $50.0 \%$ ) than in urban areas (38.7\%), which showed a statistically significant difference $(\mathrm{p}=0.024 ;$ Table 1$)$

\section{The prevalence of periodontitis based on oral health-related characteristics}

Examination of the prevalence of periodontitis based on the research subjects'oral health-related characteristics showed the following: by average number of daily brushings, periodontitis was most prevalent among subjects who brushed less than once per day (49.5\% twice $40.5 \%$, three times or more $37.9 \%$; $=0.040$ ) by oral hygiene product use, periodontitis was highly prevalent 
Table 1. Comparison of Periodontitis Prevalence by General Characteristics $(n=1,021)$

\begin{tabular}{lrrc}
\hline \multicolumn{1}{c}{ Variable } & Normal & Periodontitis & p-value \\
\hline Gender & & & 0.490 \\
Male & $251(53.5)$ & $218(46.5)$ & \\
Female & $324(58.5)$ & $228(41.5)$ & \\
Education & & & 0.064 \\
$\quad$ Elementary school & $368(55.8)$ & $292(44.2)$ & \\
$\quad$ Middle school & $74(48.5)$ & $62(51.5)$ & \\
High school & $76(61.3)$ & $64(38.7)$ & \\
University or over & $51(72.7)$ & $23(27.3)$ & \\
Income & & & 0.965 \\
Low & $136(57.2)$ & $115(42.8)$ & \\
Middle-low & $123(55.6)$ & $102(44.4)$ & \\
Middle-high & $150(57.9)$ & $111(42.1)$ & \\
$\quad$ High & $157(58.3)$ & $110(41.7)$ & \\
Living area & & & 0.024 \\
$\quad$ Urban & $427(61.3)$ & $298(38.7)$ & \\
$\quad$ Rural & $148(50.0)$ & $148(50.0)$ & \\
\hline
\end{tabular}

Values are presented as $\mathrm{n}(\%)$.

Table 2. Comparison of Periodontitis Prevalence by Oral Health Related Characteristics $(n=1,021)$

\begin{tabular}{|c|c|c|c|}
\hline Variable & Normal & Periodontitis & p-value \\
\hline \multicolumn{3}{|c|}{ The frequency of tooth-brushing } & 0.040 \\
\hline Less or once & $147(50.5)$ & $144(49.5)$ & \\
\hline Twice & $263(59.5)$ & $198(40.5)$ & \\
\hline Three time or more & $165(62.1)$ & 104 (37.9) & \\
\hline $\begin{array}{l}\text { Use of oral hygiene } \\
\text { devices }\end{array}$ & & & 0.042 \\
\hline Yes & $89(67.1)$ & 49 (32.9) & \\
\hline No & $485(56.1)$ & 397 (43.9) & \\
\hline Oral health check-up & & & 0.788 \\
\hline Yes & $91(58.7)$ & $64(41.3)$ & \\
\hline No & $484(57.2)$ & $382(42.8)$ & \\
\hline Missing tooth & & & 0.149 \\
\hline Yes & $303(54.9)$ & $264(45.1)$ & \\
\hline No & $272(60.3)$ & $182(39.7)$ & \\
\hline
\end{tabular}

Values are presented as $\mathrm{n}(\%)$.

among those who did not use oral hygiene products (43.9\%, $\mathrm{p}=0.042$ ). By dental checkup, the prevalence of periodontitis was high among those who had not had any dental checkups within the past year $(42.8 \%)$; by tooth loss, the prevalence of periodontitis was high among subjects who reported to have lost teeth (45.1\%); however, the difference was not statistically significant (Table 2).
Table 3. Comparison of Periodontitis Prevalence by Health Related Characteristics $(n=1,021)$

\begin{tabular}{lrrc}
\hline \multicolumn{1}{c}{ Variable } & Normal & Periodontitis & p-value \\
\hline Drinking & & & 0.383 \\
$\quad$ Yes & $396(55.2)$ & $321(44.8)$ & \\
$\quad$ No & $172(60.0)$ & $120(40.0)$ & \\
Smoking experience & & & 0.841 \\
$\quad$ Current smoking & $71(55.8)$ & $64(44.2)$ & \\
Past smoking & $161(59.1)$ & $137(40.9)$ & \\
No & $336(57.0)$ & $240(43.0)$ & \\
BMI & & & 0.484 \\
Underwight & $17(64.4)$ & $9(35.6)$ & \\
Normal & $227(57.6)$ & $167(42.4)$ & \\
Overwight & $147(60.6)$ & $109(39.4)$ & \\
Obesity & $184(53.6)$ & $159(46.4)$ & \\
Regular pysical activity & & & 0.684 \\
Yes & $108(55.1)$ & $96(44.9)$ & \\
$\quad$ No & $400(56.8)$ & $312(43.2)$ & \\
Walking practice & & & 0.489 \\
$\quad$ Yes & $386(57.3)$ & $303(42.7)$ & \\
$\quad$ No & $121(60.6)$ & $90(39.4)$ & \\
\hline
\end{tabular}

Values are presented as $\mathrm{n}(\%)$.

3. The prevalence of periodontitis based on healthrelated characteristics

Examination of the prevalence of periodontitis based on the research subjects' health-related characteristics showed the following: by alcohol consumption status, the prevalence of periodontitis was high among subjects who drank alcohol (44.8\%); by smoking status, the prevalence was high in the order of current smokers $(44.2 \%)$, non-smokers $(43.0 \%)$, and previous smokers $(40.9 \%)$; by body mass index, the prevalence was high in the order of obesity (46.4\%), normal weight (42.4\%), overweight $(39.4 \%)$, and underweight (35.6\%). By level of activity, the prevalence was high among subjects who reported engaging in a moderate level of exercise (44.9\%), followed by those who walked (42.7\%) (Table 3).

\section{The prevalence of periodontitis based on blood levels of vitamin $D$ and bone mineral density}

Examination of the prevalence of periodontitis based on the research subjects' blood vitamin D level showed the following: the prevalence of periodontitis was highest among subjects with vitamin D levels below $13.23 \mathrm{ng} / \mathrm{ml}$ (49.4\%), followed by levels between $16.96 \sim 21.58 \mathrm{ng} / \mathrm{ml}$ 
(44.9\%), above $21.59 \mathrm{ng} / \mathrm{ml}(39.2 \%)$, and between $13.24 \sim$ $16.95 \mathrm{ng} / \mathrm{ml}(38.8 \%)$. There was no statistically significant difference. The prevalence of periodontitis based on bone mineral density was highest among subjects with a normal range $(44.5 \%)$, followed by those with osteoporosis $(41.7 \%)$ and osteopenia $(40.1 \%)$. There was no statistically significant difference (Table 4).

\section{Blood levels of vitamin D level and their correlation to periodontitis}

To examine the correlation between blood levels of vitamin $\mathrm{D}$ and periodontitis, logistic regression analysis was performed by adjusting for disruption variables separately. Model 1 was adjusted for general characteristics; Model 2 for oral health-related characteristics; Model 3 for health-related characteristics; Model 4 for general characteristics and health-related characteristics; Model 5 for health-related and oral health-related characteristics; Model 6 for general, health-related, and oral health-related characteristics; and Model 7 for general, health-related, and oral health-related characteristics, as well as bone mineral density. When the disruption variables were not adjusted, the risk for periodontitis decreased with reduced blood vitamin D levels; however, the difference was not statistically significant. In Model 3, the risk for periodontitis in the Q1 group increased 1.68 times (1.00 2.83) compared to that in the Q4 group. The difference was statistically significant ( $p=0.047)$. In Model

Table 4. Comparison of Periodontitis Prevalence by Vitamin D Level and Bone Mineral Density

\begin{tabular}{lrrr}
\hline \multicolumn{1}{c}{ Variable } & Normal & Periodontitis & p-value \\
\hline Vitamin D level $^{\mathrm{a}}$ & & & 0.208 \\
Q1 & $113(50.6)$ & $109(49.4)$ & \\
Q2 & $124(61.2)$ & $96(38.8)$ & \\
Q3 & $132(55.1)$ & $102(44.9)$ & \\
Q4 & $206(60.8)$ & $139(39.2)$ & \\
Bone mineral density & & & 0.552 \\
Normal & $318(55.5)$ & $258(44.5)$ & \\
Osteopenia & $226(59.9)$ & $165(40.1)$ & \\
Osteoporosis & $31(58.3)$ & $23(41.7)$ & \\
\hline
\end{tabular}

Values are presented as $\mathrm{n}(\%)$.

${ }^{\mathrm{a} Q 4}$ : > $21.59 \mathrm{ng} / \mathrm{ml}, \mathrm{Q} 3: 16.96 \sim 21.58 \mathrm{ng} / \mathrm{ml}, \mathrm{Q} 2: 13.24 \sim 16.95$ $\mathrm{ng} / \mathrm{ml}, \mathrm{Q} 1: \leq 13.23 \mathrm{ng} / \mathrm{ml}$. ${ }^{\mathrm{b}}$ Normal: T-score $\geq-1.0$, osteopenia: $-2.5<\mathrm{T}$-score $<-1.0$, osteoporosis: T-score $\leq-2.5$.

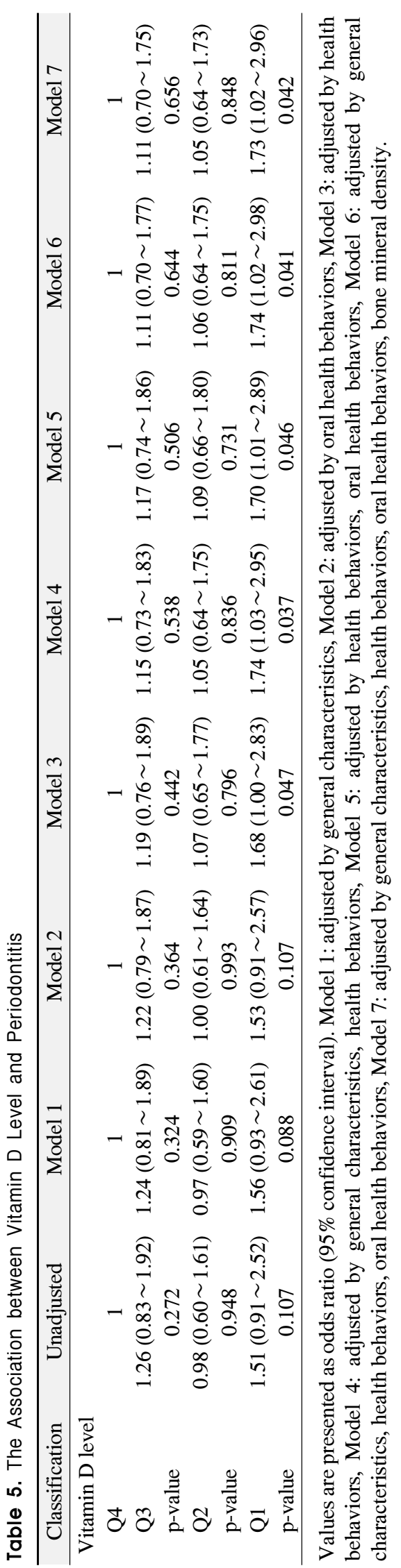


4, the risk for periodontitis in the Q1 group increased 1.74 times $(1.03 \sim 2.95)$ compared to that in the Q4 group, astatistically significant difference $(\mathrm{p}=0.037)$. In Model 5, the risk for periodontitis in the Q1 group increased 1.70 times (1.01 2.89) compared to that in the Q4 group, a statistically significant difference $(\mathrm{p}=0.046)$. In Model 6, the risk for periodontitis in the Q1 group increased 1.74 times $(1.02 \sim 2.98)$ compared to that in the Q4 group, a statistically significant difference $(p=0.041)$. Finally, in Model 7, the risk for periodontitis in the Q1 group increased 1.73 times $(1.02 \sim 2.96)$ compared to that in the Q4 group, a statistically significant difference $(p=0.042$, Table 5).

\section{Discussion}

Periodontal disease, which is caused by bacterial infection and lowered immunity of the host, progresses to alveolar bone resorption and periodontal tissue loss ${ }^{23)}$. The prevalence of periodontal disease increases with age.

According to Kim and Lee ${ }^{24)}, 57.9 \%$ of the elderly population perceives their dental health to be poor. According to Jang and $\mathrm{Nam}^{25)}$, the number of sextants with periodontal pockets increases with age.

Nutritional elements are related not only to oral health such as dental caries or periodontal diseases but also to systematic diseases such as diabetes, cardiovascular disease, and osteoporosis ${ }^{26)}$. In particular, vitamin D helps with bone growth and retention, maintains the homeostasis of calcium in the bone, and controls immune system with a natural inoculation effect ${ }^{9)}$. The present study presumed that a low level of vitamin D in the blood increases the prevalence of periodontitis and investigated the correlation of periodontitis to blood levels of vitamin D level in elderly people over 65 years of age using data on bone mineral density, blood vitamin D level, and periodontitis from the 2010 KNHANES. In this study, the vitamin D levels were divided into quartiles: Q1 had vitamin D levels below $13.23 \mathrm{ng} / \mathrm{ml}$; Q2 had levels between 13.24 16.95 ng/ml; Q3 had levels between 16.96 21.58 ng/ml; and Q4 had levels above $21.59 \mathrm{ng} / \mathrm{ml}$. Logistic regression analysis was performed by adjusting for disruption variables in order to examine the blood vitamin $\mathrm{D}$ level in elderly people assessed by the KNHANES and to examine the size and significance of correlation of periodontitis with blood levels of vitamin D based on the vitamin D level problem and previous study findings.

Examination of blood levels of vitamin D revealed the following: $21.1 \%$ of subjects had vitamin D levels below $13.23 \mathrm{ng} / \mathrm{ml}, 22.1 \%$ had levels between $13.24 \sim 16.95$ $\mathrm{ng} / \mathrm{ml}, 23.2 \%$ had levels between $16.96 \sim 21.58 \mathrm{ng} / \mathrm{ml}$, and $33.6 \%$ had levels above $21.59 \mathrm{ng} / \mathrm{ml}$. According to the data from the United States (US) National Health and Nutrition Examination Survey (NHANES), 46\% of American adults had vitamin D deficiency, with levels below $20 \mathrm{ng} / \mathrm{ml}^{27)}$. As for South Korea, the 2010 KNHANES reported that $65.9 \%$ of adults over 20 years were vitamin $\mathrm{D}$ deficient. In addition, $7.4 \%$ of subjects had vitamin D levels below $10 \mathrm{ng} / \mathrm{ml}$, which is a seriously deficient state. Compared to the US, the vitamin D levels among the Korean people are a markedly low. As for the elderly, $44.9 \%$ of men and $76.6 \%$ of women had Vitamin D levels below $20 \mathrm{ng} / \mathrm{ml}$. The reason for such a high percentage in elderly women is that the rate of cholesterol transformation to estrogen decreases after menopause, which leads to increased cholesterol levels in the blood ${ }^{28)}$. In return, increased fat tissues lower levels of vitamin D in the blood ${ }^{29)}$; therefore, it is speculated that women have lower blood vitamin D levels than men.

Examination of periodontitis status based on blood levels of vitamin D showed that the group with high vitamin D level (Q4) had a higher prevalence of periodontitis compared to that of the group with a low vitamin D level (Q1), but the difference was not statistically significant. This finding is consistent with the report by Lee and $\mathrm{Roh}^{30)}$ that the relationship between blood levels of vitamin D among men in their 50s and periodontal disease was not significant. However, this result conflicts with the finding by Jimenez et al. ${ }^{31)}$ who reported that the risk for periodontal disease decreased by $9 \%$ with a 10 $\mathrm{nmol} / \mathrm{L}$ increase in blood vitamin $\mathrm{D}$ among men between 40 and 75 years of age. It is also contrary to the finding of Dietrich et al. ${ }^{13)}$, who reported that the group of subjects over 50 years with higher blood vitamin D level has less attachment tissue loss. Such conflicting results may be explained by the following. Previous studies employed 
different measurement criteria for blood levels of vitamin D level, and Jimenez et al. ${ }^{31)}$ determined the periodontitis status based solely on the history of being diagnosed with periodontitis. Considering that the correlation between vitamin D level and attachment tissue loss among women in the study by Dietrich et al. ${ }^{13)}$ was in significant, sex difference may explain the conflicting results.

To examine the size and significance of the correlation between periodontitis and blood level of vitamin $\mathrm{D}$, logistics regression analysis was performed by differently adjusting for each disruption variable. When health-related characteristics were adjusted, the Q1 group's risk for periodontitis was 1.68 times $(1.00 \sim 2.83)$ higher than that of the Q4 group; when general and health-related characteristics were adjusted, the Q1 group's risk increased by 1.74 times $(1.03 \sim 2.95)$. When health-related and oral health-related characteristics were adjusted, the risk increased by 1.70 times $(1.01 \sim 2.89)$; when general, oral health-related, and health-related characteristics were adjusted, the risk increased by 1.74 times $(1.02 \sim 2.98)$. When all disruption variables were adjusted, the $\mathrm{Q} 1$ group's risk for periodontitis increased by 1.73 times (1.02 2.96), which demonstrates that a low level of vitamin $\mathrm{D}$ in the blood increases the risk of periodontitis. Meanwhile, there was no significant correlation between periodontitis and blood levels of vitamin D among research subjects under 64 years of age, although the details are not presented in this paper. The study findings matched those of Dietrich et al. $^{32)}$ that a higher level of vitamin D in the blood was related to a lower level of attachment tissue loss among men and women over 50 years of age, while there was no significant correlation between attachment tissue loss and blood vitamin D level among subjects ages between 20 and 49 years. Considering the fact that $58.5 \%$ of the research subjects were elderly women, these findings can be interpreted as the result of the skin's decreased capability for vitamin D synthesis with age as the skin becomes thinner and the number of keratinocytes or fibrocytes reduces while vitamin D levels in the blood decrease due to increased serum cholesterol levels ${ }^{30}$.

Dietrich et al. ${ }^{13)}$ reported that higher blood levels of vitamin D contribute to reduced gingival bleeding. According to the study, the presence of gingival bleeding indicates a gingival infection; the consumption of optimal levels of vitamin D and calcium can prevent tooth loss among elderly people because the progression of gingivitis can lead to periodontal tissue destruction ${ }^{33)}$. Findings from previous studies suggest an optimal blood vitamin D level $36 \sim 40 \mathrm{ng} / \mathrm{ml}$ for the prevention of periodontitis $^{34)}$.

The present study is limited in that other factors influencing blood levels of vitamin D such as exercise, calcium intake, hormone supplementation, and systematic disease were not considered, and that the correlation to periodontitis cannot be interpreted in terms of a causal relationship due to the cross-sectional nature of the study design. To rectify these limitations, epidemiological research needs to be conducted in a follow-up clinical study that can evaluate the factors influencing blood levels of vitamin $\mathrm{D}$ and the dose-response relationship to periodontitis and identify the causal relationships. Despite these limitations, the present study is significant in that it utilized highly reliable data from the KNHANES, which represents the entire population. The results of this study provide evidence for the necessity of vitamin $\mathrm{D}$ control for periodontitis prevention and treatment by presenting the correlation between blood levels of vitamin D and periodontitis

\section{References}

1. Ministry of Health and Welfare: Korea health statistics: 2014 Korea national health and nutrition examination survey. Available from: https://knhanes.cdc.go.kr/knhanes/sub04/ sub04_03.do?classType=7(2014).

2. Holick MF: Vitamin D deficiency. N Engl J Med 357: 266-281, 2007.

3. Jimi E, Nakamura I, Amano H, et al.: Osteoclast function is activated by osteoblastic cells through a mechanism involving cell-to-cell contact. Endocrinology 137: 2187-2190, 1996.

4. Hildebolt CF: Osteoporosis and oral bone loss. Dentomaxillofac Radiol 26: 3-15, 1997.

5. Payne JB, Reinhardt RA, Nummikoski PV, Patil KD: Longitudinal alveolar bone loss in postmenopausal osteoporotic/osteopenic women. Osteoporos Int 10: 34-40, 1999. 
6. Passos Jde S, Gomes-Filho IS, Vianna MI, et al.: Outcome measurements in studies on the association between osteoporosis and periodontal disease. J Periodontol 81: 1773-1780, 2010.

7. Taguchi A, Suei Y, Ohtsuka M, Otani K, Tanimoto K, Hollender LG: Relationship between bone mineral density and tooth loss in elderly Japanese women. Dentomaxillofac Radiol 28: 219-223, 1999.

8. Song HJ, Lee DH: Relationship between bone mineral density and the remaining teeth in Korean adults. J Korean Soc Dent Hgy 15: 63-71, 2015.

9. Han EY, Rhyu IC, Lee YM, et al.: Relationships between osteoporosis, alveolar bone density and periodontal disease in postmenopausal women. J Korean Acad Periodontol 31: 565-571, 2001.

10. Yoshihara A, Seida Y, Hanada N, Miyazaki H: A longitudinal study of the relationship between periodontal disease and bone mineral density in community-dwelling older adults. J Clin Periodontol 31: 680-684, 2004.

11. Tezal M, Wactawski-Wende J, Grossi SG, Dmochowski J, Genco RJ: Periodontal disease and the incidence of tooth loss in postmenopausal women. J Periodontol 76: 1123-1128, 2005.

12. Hewison M: Vitamin D and the intracrinology of innate immunity. Mol Cell Endocrinol 321: 103-111, 2010.

13. Dietrich T, Nunn M, Dawson-Hughes B, Bischoff-Ferrari HA: Association between serum concentrations of 25-hydroxyvitamin D and gingival inflammation. Am J Clin Nutr 82: 575-580, 2005.

14. McMahon L, Schwartz K, Yilmaz O, Brown E, Ryan LK, Diamond G: Vitamin D-mediated induction of innate immunity in gingival epithelial cells. Infect Immun 79: 2250-2256, 2011.

15. Shils ME, Olson JA, Shike M, Ross AC: Modern nutrition in health and disease: bone biology in health and disease. 9th ed. Williams \& Wilkins, Pennsylvania, pp.1314-1324, 1999.

16. Kim YS, Yoon BK, Kim JY, et al.: Effect of hormone replacement therapy on bone mineral density in Korean postmenopausal women over age of 60. J Menopausal Med 13: 114-122, 2007.

17. Zittermann A: Vitamin D and disease prevention with special reference to cardiovascular disease. Prog Biophys Mol Biol 92: 39-48, 2006.
18. Parfitt AM, Gallaher JC, Heaney RP, Johnston CC, Neer R, Whedon CD: Vitamin D and bone health in the elderly. Am J Clin Nutr 36(5 Suppl): 1014-1031, 1982.

19. Bullon P, Goberna B, Guerrero JM, Segura JJ, Perez-Cano R, Martinez-Sahuquillo A: Serum, saliva, and gingival crevicular fluid osteocalcin: their relation to periodontal status and bone mineral density in postmenopausal women. J Periodontol 76: 513-519, 2005.

20. Dawson-Hughes B, Harris SS, Krall EA, Dallal GE: Effect of calcium and vitamin D supplementation on bone density in men and women 65 years of age or older. N Engl J Med 337: 670-676, 1997.

21. World Health Organization: Assessment of fracture risk and its application to screening for postmenopausal osteoporosis: report of a WHO study group. World Health Organization, Geneva, pp.1-129, 1994.

22. Cutress TW, Ainamo J, Sardo-Infirri J: The community periodontal index of treatment needs (CPITN) procedure for population groups and individuals. Int Dent J 37: 222-233, 1987.

23. Kim KM, Kim YJ, Chung HJ: Osteoporotic condition in postmenopausal women with periodontitis. J Korean Periodontol Implant Sci 32: 225-234, 2002.

24. Kim MJ, Lee HK: Relationship of dental health assessment to the number of existing permanent tooth in senior citizens visited a dental hospital or clinic from some regions. J Dent Hyg Sci 7: 161-166, 2007.

25. Jang BJ, Nam IS: Relationship between smoking, drinking and periodontal pocket formation of patient was visited at college scaling center. J Dent Hyg Sci 10: 117-122, 2010.

26. Palacios C, Joshipura K, Willett W: Nutrition and health: guidelines for dental practitioners. Oral Dis 15: 369-381, 2009.

27. Forrest KY, Stuhldreher WL: Prevalence and correlates of vitamin D deficiency in US adults. Nutr Res 31: 48-54, 2011.

28. Broussard DL, Magnus JH: Coronary heart disease risk and bone mineral density among U.S. women and men. J Womens Health (Larchmt) 17: 479-490, 2008.

29. Snijder MB, van Dam RM, Visser M, et al.: Adiposity in relation to vitamin $\mathrm{D}$ status and parathyroid hormone levels: a population-based study in older men and women. J Clin Endocrinol Metab 90: 4119-4123, 2005.

30. Lee SH, Roh SC: Vitamin D level in blood of menopausal 
women over 50 and the relation with the proportion requiring dental scaling. J Dent Hyg 13: 393-402, 2013.

31. Jimenez M, Giovannucci E, Krall Kaye E, Joshipura KJ, Dietrich T: Predicted vitamin D status and incidence of tooth loss and periodontitis. Public Health Nutr 17: 844-852, 2014.

32. Dietrich T, Joshipura KJ, Dawson-Hughes B, Bischoff-Ferrari HA: Association between serum concentrations of 25-hydroxyvitamin D3 and periodontal disease in the US population. Am J Clin Nutr 80: 108-113, 2004

33. Krall EA, Wehler C, Garcia RI, Harris SS, Dawson-Hughes B: Calcium and vitamin D supplements reduce tooth loss in the elderly. Am J Med 111: 452-456, 2001.

34. Bischoff-Ferrari HA, Giovannucci E, Willett WC, Dietrich T, Dawson-Hughes B: Estimation of optimal serum concentrations of 25-hydroxyvitamin D for multiple health outcomes. Am J Clin Nutr 84: 18-28, 2006. 